\title{
CHARACTERIZING THE NONTHERMAL EMISSION OF CASSIOPEIA A
}

\author{
E. A. Helder ${ }^{1}$ AND J. VINK ${ }^{1}$ \\ Received 2008 March 11; accepted 2008 June 19
}

\begin{abstract}
We report on our analysis of the 1 Ms Chandra observation of the supernova remnant Cas A in order to localize, characterize, and quantify the nonthermal X-ray emission. More specifically, we investigated whether the X-ray synchrotron emission from the inside of the remnant is from the outward shock, but projected toward the inner ring, or from the inner shell. We tackled this problem by employing a Lucy-Richardson deconvolution technique and measuring spectral indices in the $4.2-6 \mathrm{keV}$ band. We show that most of the continuum emission is coming from an inner ring that coincides with the previously reported location of the reverse shock. This inner ring also includes filaments whose X-ray emission has been found to be dominated by X-ray synchrotron emission. The X-ray emission from these filaments, both at the forward shock and from the inner ring, have relatively hard spectra with spectral index $>-3.1$. The regions emitting hard X-ray continuum contribute about $54 \%$ of the total X-ray emission in the $4.2-6 \mathrm{keV}$. This is lower than that suggested by extrapolating the hard X-ray spectrum as measured by BeppoSAX PDS and INTEGRAL. This can be reconciled by assuming a gradual steepening of the spectrum toward higher energies. We argue that the X-ray synchrotron emission is mainly coming from the western part of the reverse shock. The reverse shock in the west is almost at rest in our observation frame, corresponding to a relatively high reverse shock velocity of $\sim 6000 \mathrm{~km} \mathrm{~s}^{-1}$ in the frame of the freely expanding ejecta.
\end{abstract}

Subject headings: acceleration of particles - ISM: individual (Cassiopeia A) radiation mechanisms: nonthermal — shock waves - supernova remnants

Online material: color figures

\section{INTRODUCTION}

Supernova remnants (SNRs) are the main candidates for producing Galactic cosmic rays, with energies at least up to the socalled knee of the cosmic ray spectrum at $\sim 3 \times 10^{15} \mathrm{eV}$. The first direct evidence for this is the detection of X-ray synchrotron emission caused by $\sim 10^{14} \mathrm{eV}$ electrons (first established for SN 1006; Koyama et al. 1995). Since the energy of electrons suffers from radiation losses, this might indicate even higher energies for ions. Moreover, hard X-ray tails up to $80 \mathrm{keV}$ have been discovered for several Galactic SNRs (Allen et al. 1999). This has been contributed to either nonthermal bremsstrahlung (Laming 2001) or to synchrotron radiation (Allen et al. 1997). In recent years, additional direct evidence for efficient cosmic-ray acceleration has come from detection of $\mathrm{TeV} \gamma$-rays for several SNRs by the High Energy Gamma-Ray Astronomy (HEGRA) experiment, the High Energy Spectroscopic System (H.E.S.S.; see, e.g., Aharonian et al. 2004), and MAGIC (Albert et al. 2007). The $\gamma$-ray emission is either caused by inverse Compton scattering by the same electrons that cause X-ray synchrotron emission, or by pion production caused by collisions of accelerated ions with the background plasma.

Cassiopeia A (Cas A) is one of the supernova remnants with a hard X-ray tail (The et al. 1996) and has recently also been detected in $\gamma$-rays (Aharonian et al. 2001; Albert et al. 2007). This remnant was until recently ${ }^{2}$ the youngest known supernova remnant in the Galaxy; its supernova was probably around 1671 (Thorstensen et al. 2001). In 2001 Chandra detected thin, X-ray synchrotron emitting filaments at the forward shock of the remnant (Fig. 1; see also Gotthelf et al. 2001). This implies the presence of electrons with energies of $\sim 10^{13} \mathrm{eV}$ for the magnetic fields in Cas A, estimated to be $0.1-0.6 \mathrm{mG}$ (Vink \& Laming 2003; Berezhko \&

\footnotetext{
1 Astronomical Institute Utrecht, Utrecht University, P.O. Box 80000, NL -3508 TA Utrecht, The Netherlands.

${ }^{2}$ Recent expansion measurements of G1.9+0.3 show an age for this remnant of around 100 years (Green et al. 2008; Reynolds et al. 2008).
}

Völk 2004). These synchrotron rims can be understood in the context of diffusive shock acceleration and synchrotron cooling downstream of the shock.

In addition, the Chandra image shows thin filaments at the inside of the remnant. Some of these inner filaments show a featureless spectrum (Fig. 2). Hughes et al. (2000) identified one of the inner filaments at the west side of the remnant ("region D") as being the projected forward shock, based on its featureless spectrum. DeLaney et al. (2004) found that the kinematics of the inner filaments, which they interpreted as projected forward shock filaments, are different from the forward shock; they have a lower velocity.

Diffusive shock acceleration is a process that accelerates cosmic rays at a shock front (for a review, see Malkov \& Drury 2001). This mechanism accelerates charged particles of sufficient energy, which scatter on turbulent magnetic fields/plasma waves on both sides of the shock front. Each time the shock front is crossed, the particle gains energy due to the difference in plasma velocity between both sides of the shock front. The higher the difference in the velocities, the more energy that is gained in one iteration, and the higher the magnetic field and magnetic field turbulence, the more often particles cross the shock front. Since at the location where efficient particle acceleration takes place recently accelerated electrons are present, these locations show X-ray synchrotron radiation. However, farther downstream from the shock front, synchrotron losses result in lower maximum energies of the synchrotron radiation.

The synchrotron spectrum can be approximated over a large range in frequencies with a power law in flux density: $F_{\nu} \propto \nu^{-\alpha}$ with an index $(\alpha)$ related to the power-law index of the electron distribution $(p)$ as $\alpha=(p-1) / 2$. In what follows, we use index $\Gamma$, which refers to the number density index $\Gamma=-(\alpha+1)$ and $n(E) \propto E^{\Gamma}$. Near the maximum electron energies, the electron spectrum has an exponential cutoff, but the resulting synchrotron spectrum cuts off less abruptly, roughly as $\exp \left[-\left(\nu / \nu_{\max }\right)^{1 / 2}\right]$ (Zirakashvili \& Aharonian 2007). In contrast, the other important 


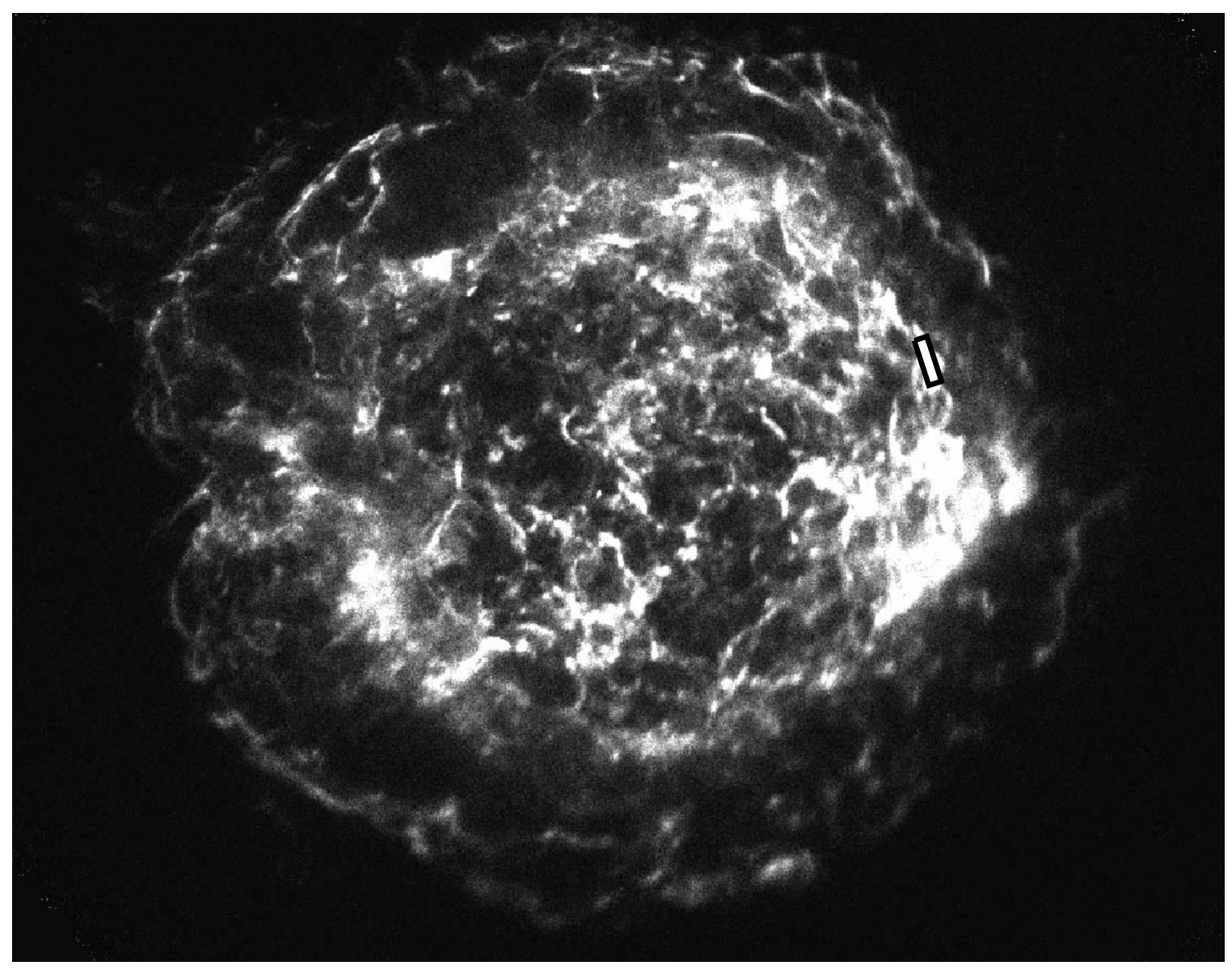

FIg. 1.- The $1 \mathrm{Ms} 4-6 \mathrm{keV}$ continuum image of Cas A, obtained with Chandra. The rectangle indicates region D in Hughes et al. (2000). [See the electronic edition of the Journal for a color version of this figure.]

continuum emission process, thermal bremsstrahlung, has an exponential cutoff $[\propto \exp (-h \nu / k T)]$. In Cas A, the plasma temperature ranges between 0.6 and $3.6 \mathrm{keV}$ (Yang et al. 2007). One should take into account that these temperatures originate from a thermal model. If a partly nonthermal spectrum is fitted with a thermal model, the fitted temperature tends to increase with respect

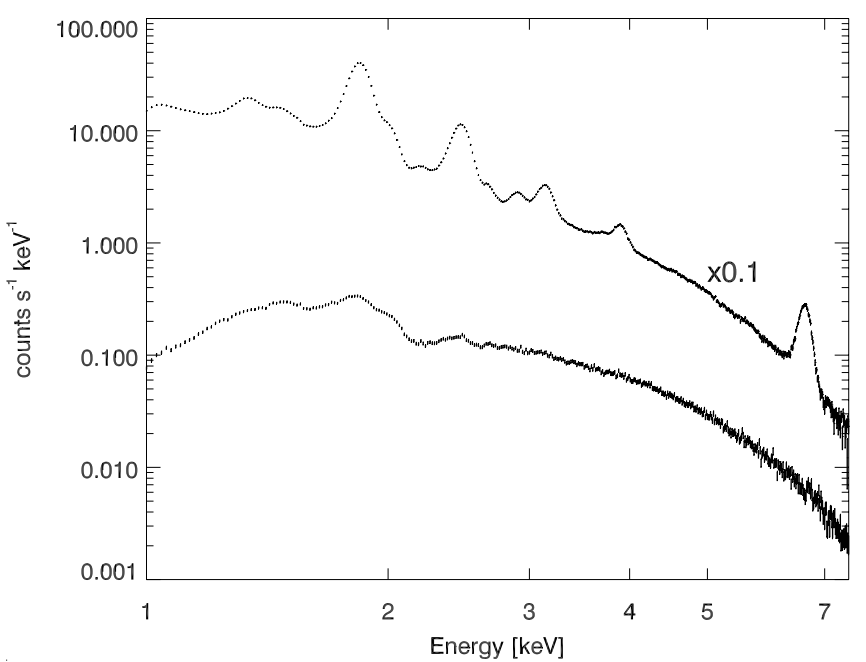

FIG. 2.- Spectrum of Cas A as observed by Chandra. Below is the spectrum of the featureless filament (D) described by Hughes et al. (2000), extracted from the megasecond observation; above is a spectrum of the whole remnant of one single observation (obsID 4638), multiplied by 0.1 . to the real temperature of the plasma. For a thermal bremsstrahlung spectrum with a temperature of $3.5 \mathrm{keV}$, the power-law index between 4.2 and $6.0 \mathrm{keV}$ is -2.8 . For a synchrotron spectrum at the forward shock of Cas A, we typically measure a power-law index of -2.1 . We therefore expect the bremsstrahlung continuum to be steeper than the synchrotron continuum in the $4-6 \mathrm{keV}$ continuum band.

In this paper we investigate the shape of the continuum spectrum and its spatial distribution in order to address several questions pertaining to the shock acceleration in Cas A: What is the location of the X-ray synchrotron filaments? What fraction of the overall $\mathrm{X}$-ray continuum is thermal and what fraction is nonthermal? And what are the implications for the hard X-ray emission, above $10 \mathrm{keV}$ ? We do this by analyzing the Chandra megasecond observation of Cas A.

\section{THE USED DATA}

Chandra observed Cas A for $1 \mathrm{Ms}$ in 2004 from February to May (Hwang et al. 2004) with the ACIS S3. For extracting the images, we use the CIAO package, version 3.4. To align the separate pointings, the central compact object is taken as a reference point. We mostly concentrate on the $4.2-6 \mathrm{keV}$ energy band, but we also extracted an image in the line of $\mathrm{Si}$ XIII $\mathrm{He} \alpha$ from 1.80 to $1.90 \mathrm{keV}$. We corrected this for continuum emission by subtracting the average of two images next to the Si line: in the 1.631.64 and $2.13-2.14 \mathrm{keV}$ bands.

We compared the results of our analysis of the Chandra data with a VLA radio map, made in the $6 \mathrm{~cm}$ band in 2000-2001. This map was kindly provided to us by Tracey DeLaney (Delaney et al. 
2005). The VLA image has a resolution of $0.38^{\prime \prime} \times 0.33^{\prime \prime}$, which is comparable to the Chandra telescope resolution of $0.42^{\prime \prime}$. However, the Chandra pixels size of $0.49^{\prime \prime}$ slightly undersamples the Chandra resolution.

In $\S 4$ we make use of BeppoSAX observations made in 2001 June, with an exposure time of $501 \mathrm{ks}$. The hard X-ray spectrum obtained with the PDS instrument is described in Vink et al. (2001). The data obtained with the MECS instruments were never published before, but the details of the analysis are similar to the analysis described in Vink et al. (1999) for a shorter exposure.

Finally, the INTEGRAL-ISGRI spectrum used in $\S 4$ is described in Renaud et al. (2006).

\section{SEPARATING THE FORWARD AND REVERSE SHOCK}

The surface brightness $[\Sigma(r)]$ of an optically thin object consists of the emissivity function of this object $[\epsilon(r)]$, integrated along the line of sight. For a spherically symmetric object, this integral is as follows:

$$
\Sigma(r)=2 \int_{r}^{R} \epsilon\left(r^{\prime}\right) \frac{r^{\prime}}{\sqrt{r^{\prime 2}-r^{2}}} d r^{\prime},
$$

where $R$ denotes the outer radius of this object. We do the deconvolution in cylindrical coordinates, with $\theta$ perpendicular to $r$, and $\theta=0$ is defined in the west, increasing counterclockwise. The number of counts in a sector at inner radius $r$, angular width $d \theta$ (in radians), and thickness $d r$ is

$$
C_{r}=\Sigma(r) d \theta\left[r d r+\frac{(d r)^{2}}{2}\right] .
$$

By dividing Cas A in 18 sectors of $20^{\circ}$ each, we first make surface brightness profiles of the X-ray continuum between 4.2 and $6 \mathrm{keV}$. For the surface brightness profiles, we adopt the center of expansion (Thorstensen et al. 2001): $\alpha=23^{\mathrm{h}} 23^{\mathrm{m}} 27.77^{\mathrm{s}}$ and $\delta=$ $58^{\circ} 48^{\prime} 49.4^{\prime \prime}$ (J2000.0) and take step sizes of 0.5". Furthermore, we assume spherical symmetry for each sector individually. For an example of a surface brightness profile, see the radial surface brightness profile between an angle of $10^{\circ}$ and $30^{\circ}$ in Figure 3. In this figure, we can clearly see the outer shock coming up at $160^{\prime \prime}$, as already found by Gotthelf et al. (2001). Using equations (1) and (2), we now make an emission profile in such a way that it fits the surface brightness of the outer shock (smooth solid line). As the line indicates, this thin outer shell cannot account for all the surface brightness in the center of the remnant (the line that fits the outer peak is for a small $R$ at least 15 times lower than the surface brightness profile). We also see a peak in the surface brightness at $126^{\prime \prime}$. We can identify this surface brightness peak with the featureless filament described by Hughes et al. (2000). If we now include a second emission peak at 126" (dashed line), we see that a large part of the surface brightness in the center is covered.

To go from surface brightness to emissivity we use a general deconvolution method described by Lucy (1974) and previously used on SN 1006 by Willingale et al. (1996). To test this algorithm, we simulated emission functions, convolved them into a surface brightness profile, and added Poissonian noise using the IDL routine poidev from the NASA IDL Astronomy User's Library (Landsman 1993); we made them in such a way that the peak of the number of counts in one bin of $20^{\circ} \times 0.5^{\prime \prime}$ is 11,000 , which corresponds to the peak of Figure 3 in terms of counts per $0.5^{\prime \prime} \times$ $20^{\circ}$ bin. After constructing this surface brightness profile we used the Lucy algorithm to recover the emissivity function. We stopped the deconvolution after 30 iterations. The resulting emissivity function is similar to the original one, within $10 \%$ for $R>40^{\prime \prime}$ and even within $5 \%$ for $R>75^{\prime \prime}$.

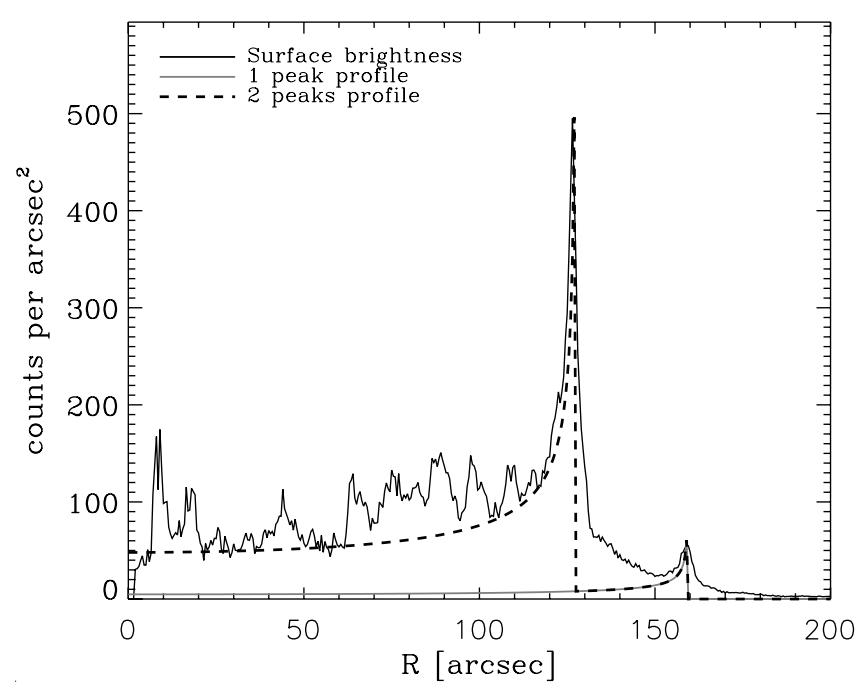

FIG. 3.- Radial surface brightness profile (smooth solid line) in the $4.2-6.0 \mathrm{keV}$ energy band at an angle of $10^{\circ}-30^{\circ}$ (including the featureless filament found by Hughes et al. 2000). The smooth solid line indicates what the profile will look like if the surface brightness is produced by an emissivity function with only a peak at the outer shock. For the dashed line, we use an emissivity function with two peaks. Note that this is not a fit, just an illustrative example. [See the electronic edition of the Journal for a color version of this figure.]

\subsection{Results of the Deconvolution}

The results of the deconvolution of the Chandra data of Cas A are given in Figure 4. We see the forward shock coming up in the $\mathrm{X}$-ray continuum at $160^{\prime \prime}$ and with a width of $10^{\prime \prime}$. What we also see is another ring inside the forward shock with a radius of $115^{\prime \prime}$ with a width of $30^{\prime \prime}$, shifted to the right by $15^{\prime \prime}$. The forward shock and this inner ring are two almost complete circles, except for the northeast region, where the jet is present. If we do the same for the radio and Si images of Cas A, we see a ring at the same location as the inner ring of the $\mathrm{X}$-ray continuum. These inner rings in radio and $\mathrm{Si}$ have in previous researches been identified as the reverse shock (Gotthelf et al. 2001).

We note that the results of the deconvolution are far from perfect; the $\chi_{\text {red }}^{2}$ of the individual fits range from 20 to 253 for $4.2-6.0 \mathrm{keV}$ and from 18 up to 1150 for the Si band. This is to be expected, since the filamentary structure is clearly inconsistent with the assumed spherical symmetry. Moreover, the X-ray Doppler maps of Cas A (Willingale et al. 2002) also show deviations from spherical symmetry. However, the fact that we can trace the inner and outer shells consistently from 18 independent deprojected sectors argues for the veracity of the two rings.

In order to estimate the total contribution of each shell to the total emissivity of Cas A in each band, we multiply the deprojected shells with the volume of the shells $\left(4 \pi R^{2} \Delta R\right)$, taking into account the relative contributions of each sector. For the continuum band in the east to south $\left(\theta \in\left[180^{\circ}, 260^{\circ}\right]\right)$, for which the overall X-ray radiation is dominated by silicon line emission, $16 \%$ comes from the outer ring and $84 \%$ comes from the inner ring. For the western part of the remnant $\left(\theta \in\left[-100^{\circ}, 20^{\circ}\right]\right)$, in which most of the filaments dominated by continuum are situated, we find that $18 \%$ of the continuum emission is due to the outer ring and $82 \%$ due to the inner ring.

\section{THERMAL BREMSSTRAHLUNG VERSUS SYNCHROTRON RADIATION}

The hard X-ray emission from Cas A is clearly nonthermal in nature (The et al. 1996; Allen et al. 1997; Favata et al. 1997). In 

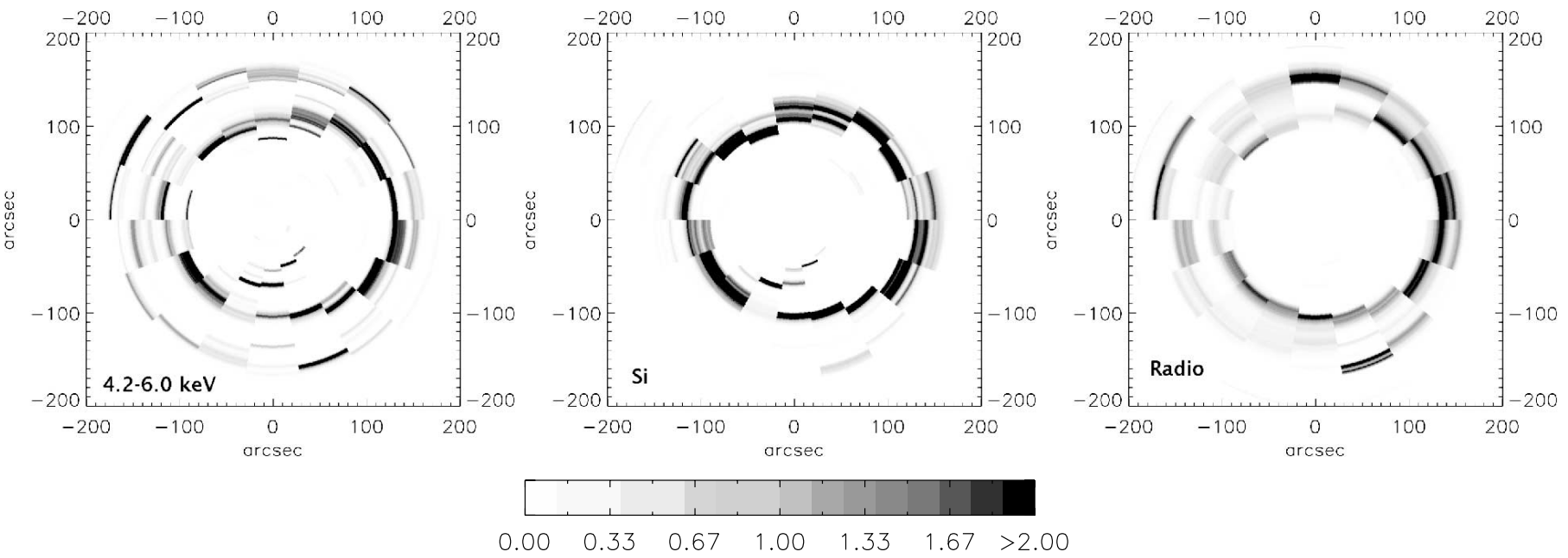

FIG. 4.-Left: Deconvolution of the 4.2-6.0 keV continuum radiation. For all the figures, the deconvolution is done in sectors; within each sector, spherical symmetry

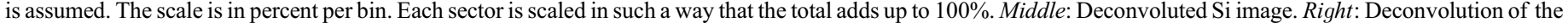
radio emission. The emissivity is multiplied with the volume of the shell to get the total emission over the whole sphere for each $d r$.

order to see how this relates to the Chandra spectrum of Cas A, we show in Figure 5 both the broadband (1-8 keV) Chandra spectrum and the hard X-ray spectrum (above $20 \mathrm{keV}$ ) as obtained with BeppoSAX PDS (Vink et al. 2001) and INTEGRAL ISGRI (Renaud et al. 2006). The model shown is fitted to the hard X-ray data only and is similar to the one used by Renaud et al., which includes the contributions of ${ }^{44} \mathrm{Ti}$ decay at 67.8 and $78.4 \mathrm{keV}$. We included absorption with $N_{\mathrm{H}}=1.3 \times 10^{22} \mathrm{~cm}^{-2}$. Of interest here are the best-fit parameters of the nonthermal component, fitted with a power law. Our best-fit parameters are $\Gamma=-3.4 \pm 0.2$, and the normalization is $3.22 \pm 1.9$ counts $\mathrm{keV}^{-1} \mathrm{~cm}^{-2} \mathrm{~s}^{-1}$ at $1 \mathrm{keV}$. We checked these results with previous results on the hard X-ray tail of the spectrum of Cas A. Rothschild \& Lingenfelter (2003) observed Cas A for $226 \mathrm{ks}$ with RXTE and fitted a power law to the HEXTE data from 20 to $200 \mathrm{keV}$. They find a power-law index of $-3.125 \pm$ 0.050 with a normalization of $2.0 \pm 0.6$ counts $\mathrm{cm}^{-2} \mathrm{~s}^{-1}$ at $1 \mathrm{keV}$.

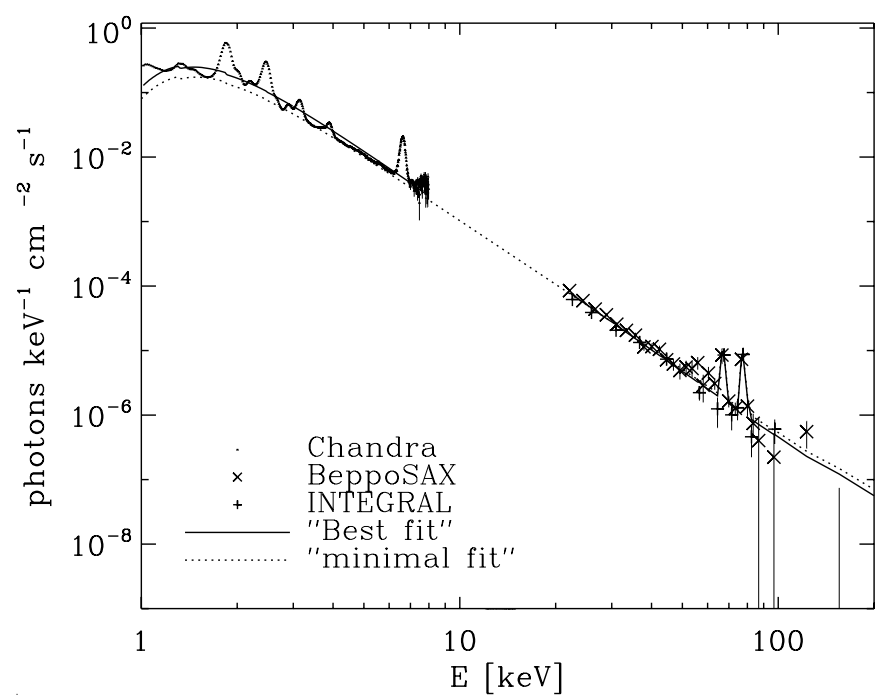

FIG. 5.-Unfolded broadband X-ray spectrum of Cas A. The solid line represents the best fit for the combined BeppoSAX PDS and INTEGRAL ISGRI data. Extrapolation of this line back to the Chandra regime fits the continuum surprisingly well. The dotted line indicates the minimal number of counts in the $4.2-6.0 \mathrm{keV}$ band, allowed within the errors of the extrapolation of the hard X-ray fit. The Chandra broadband spectrum of Cas A was made from one of the observations (obsID 4638) of the megasecond observation (Hwang et al. 2004).
We see that the BeppoSAX power law, extrapolated back to Chandra energies, fits the continuum between 2.0 and $7.0 \mathrm{keV}$ well. Within a confidence level of $90 \%$, the extrapolated power law contains a minimum of $93 \%$ of the counts in the Chandra continuum bands. The results of Rothschild \& Lingenfelter (2003) amount to a continuum flux from 4 to $6 \mathrm{keV}$ that is $28 \%$ of the continuum flux measured by Chandra. Note that if we trust the power-law continuum model, there is little room for an additional thermal component. This is surprising, since we know that the continuum in the $1-10 \mathrm{keV}$ range is at least partially due to thermal bremsstrahlung, which must accompany the copious X-ray line emission. In fact, fitting the spectrum in the $0.5-10 \mathrm{keV}$ band can be done with a pure thermal model (Vink et al. 1996; Willingale et al. 2002).

To pinpoint the different mechanisms that contribute to the continuum in the Chandra band, we fit a power law to regions of $4.9^{\prime \prime} \times 4.9^{\prime \prime}(10 \times 10$ pixels $)$ for the band between 4.2 and $6.0 \mathrm{keV}$. We use the event files of the megasecond observation, which we merge, using the central compact object as a reference point. We use one $\mathrm{ARF}^{3}$ file, made for the spectrum of Figure 5. We estimated the background contribution, using an annulus around Cas A, with $R_{\min }=205.8$ and $R_{\max }=235.2$ and a center as defined in $\S 3$. We neglected the effects of differential absorption over this small band; in the most extreme cases, the absorption varies from $1 \times 10^{22}$ to $1.7 \times 10^{22} \mathrm{~cm}^{-2}$, which has an effect of (at maximum) 0.09 in the power-law index. Fitting the continuum of the total remnant between 4.2 and $6.0 \mathrm{keV}$, using $N_{\mathrm{H}}=$ $1.3 \times 10^{22} \mathrm{~cm}^{-2}$, we found that the average index is -3.14 .

Figure 6 shows that the power-law index varies considerably inside Cas A. We see that some of the regions with hard spectra overlap with known regions of nonthermal X-ray emission. For example the forward shock region appears to have a harder power law. Also, the nonthermal filaments in the west show up in the spectral index map. However, there is also hard continuum emission in regions where there is also line emission. This is illustrated by Figures 6 and 7.

The spectral map of Figure 6 implies that if we observe Cas A at higher energies, the western part of the remnant will become more prominent. We validated this by comparing an extrapolation of our best-fit power-law spectra with the $501 \mathrm{ks}$ BeppoSAX

\footnotetext{
${ }^{3}$ Ancillary response file, which contains a description of the effective area of the instrument.
} 

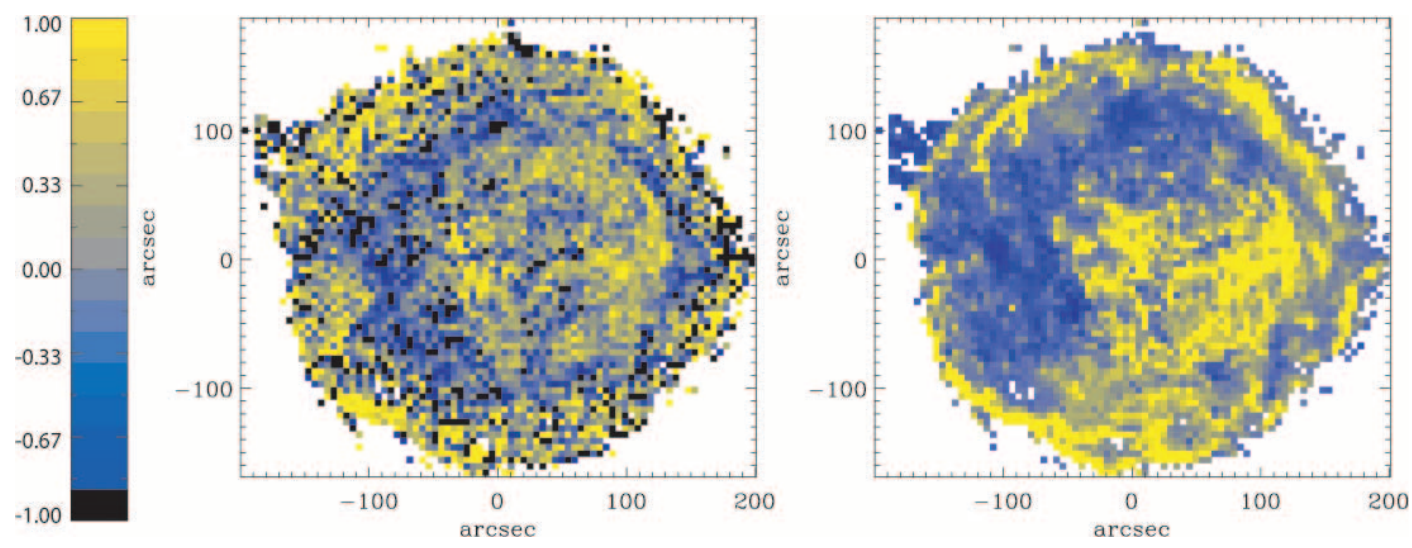

Fig. 6. - Left: Fitted power-law indices $(\Gamma)+3.1$, to show the difference between the individual fits and the overall spectrum. Darker color indicates a steeper spectrum. Right: Map of an image in the $4.2-6.0 \mathrm{keV}$ continuum bands divided by a broadband image. The lighter color means relatively more continuum and thus a harder spectrum. Note the similarities between the images: as already noted by DeLaney et al. (2004) the continuum-dominated areas tend to have a harder spectrum. Even so, not all hard spectra have a lack of line emission.

MECS observation in the $9-11 \mathrm{keV}$ band. ${ }^{4}$ We deconvolved the BeppoSAX image using the Lucy deconvolution method, following the procedure described in Vink et al. (1999). The results are shown in Figure 8; for comparison, we also show the 4-6 keV BeppoSAX image next to the Chandra image smoothed to roughly the same resolution (the extrapolation without smoothing is shown in Fig. 9). Qualitatively, the $9-11 \mathrm{keV}$ image of BeppoSAX agrees with the extrapolated Chandra image; the southeastern part of the remnant is relatively less bright, whereas the western and southern parts of the center are becoming more prominent.

Since for a bremsstrahlung continuum we expect an exponential cutoff and thus a soft spectrum, and for synchrotron radiation we expect a hard spectrum, we tentatively identify the hard spectra with synchrotron emission. Although there is a likely overlap in spectral index between thermal and nonthermal emission, we can nevertheless estimate the total contribution of nonthermal emission by noting that in Figure 6 (left), those regions that have abundant line emission (the eastern part of the shell) have power-law spec-

4 There is also an observation made by XMM-Newton of Cas A up to $15 \mathrm{keV}$ (Bleeker et al. 2001); because of errors in the exposure map of this observation, we choose to use the BeppoSAX data.

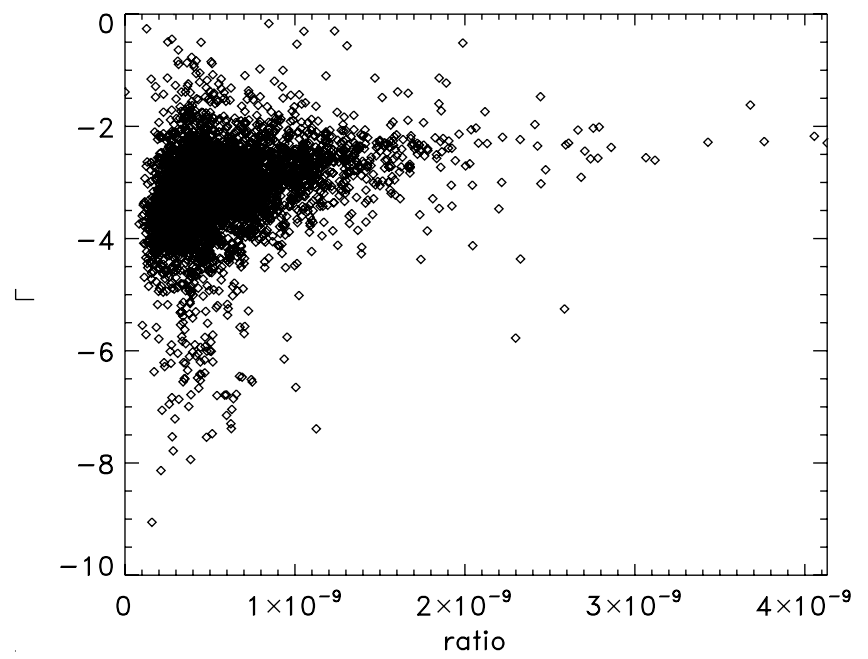

FIG. 7.- On the $x$-axis is the ratio of continuum over broadband in arbitrary units (the same units as in Fig. 6, right panel), a higher value means relatively more continuum. On the $y$-axis are the fitted power-law indices of the corresponding bins. tra steeper than -3.1 . The total flux associated with those powerlaw indices amounts to $46 \%$ of the total flux in the $4.2-6 \mathrm{keV}$ band (Fig. 10), suggesting that the other $54 \%$ of the flux is due to nonthermal radiation. This corresponds roughly to a total nonthermal flux above $4 \mathrm{keV}$ of $2.7 \times 10^{-10} \mathrm{erg} \mathrm{s}^{-1} \mathrm{~cm}^{-2}$. If we take a power-law index of -2.8 as an upper limit, this corresponds to a thermal bremsstrahlung model with $k T=3.5 \mathrm{keV}$, and the nonthermal contribution comes down to $33 \%$. This is indeed a large fraction of the total continuum emission, but not as large as the $93 \%$ suggested by extrapolation of the hard X-ray power law. The reason for this is probably that the true spectral shapes are not exactly power laws, but that they steepen at higher energies. So apparently, in the Chandra band the addition of soft thermal and

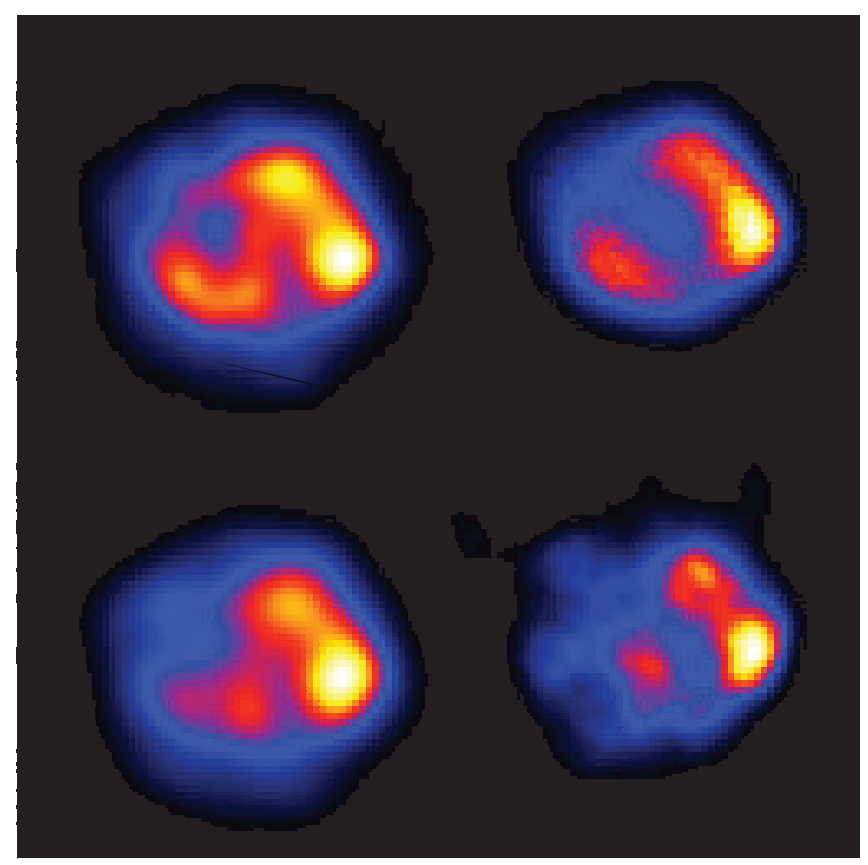

Fig. 8.-Top row: Chandra image of Cas A in the $4-6 \mathrm{keV}$ band, convolved to the BeppoSAX resolution (left) and the BeppoSAX image of Cas A in the same energy band (right). Bottom row: The left side has the image of Fig. 6, extrapolated to $9-11 \mathrm{keV}$ (Fig. 9) and convolved to the BeppoSAX resolution. The right image shows the actual BeppoSAX image in the $9-11 \mathrm{keV}$ band. The extrapolation matches qualitatively the BeppoSAX image; therefore, the power-law fits give a reasonable prediction of the hard X-ray image of Cas A. 


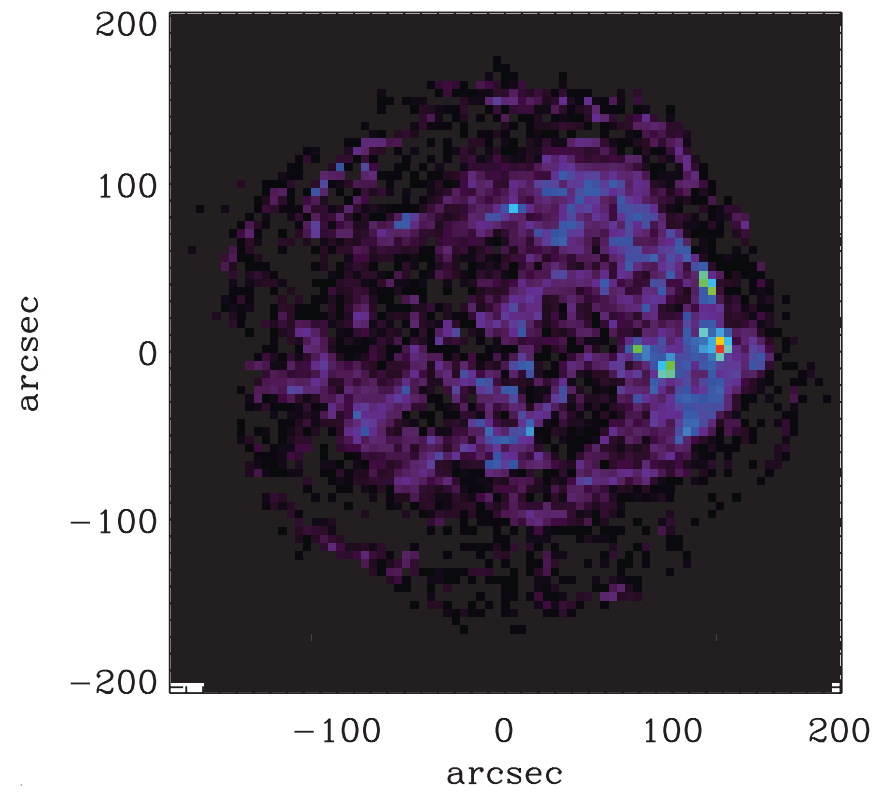

FIG. 9.- Extrapolation of the best-fit power laws from Fig. 6 to the $9-11 \mathrm{keV}$ band

hard nonthermal emission gives by coincidence almost the same power-law index as the steepening nonthermal spectra at high energies. The lack of an obvious spectral break is probably due to the variation in power-law indices for the nonthermal spectra and the variation in cutoff energies across the remnant.

\subsection{West Side of Cas $A$}

So far, we have shown that a large part of the nonthermal emission is associated with the reverse shock region and that most of this emission is coming from the western part of the remnant. This prompted us to investigate the spectrum of the western part more closely. Specifically, we were interested in whether the reverse shock in the western part shocks the less dense material. The reason is that the western region not only shows more nonthermal emission, but that there also seems to be less thermal emission. A low density of a shocked material results (apart

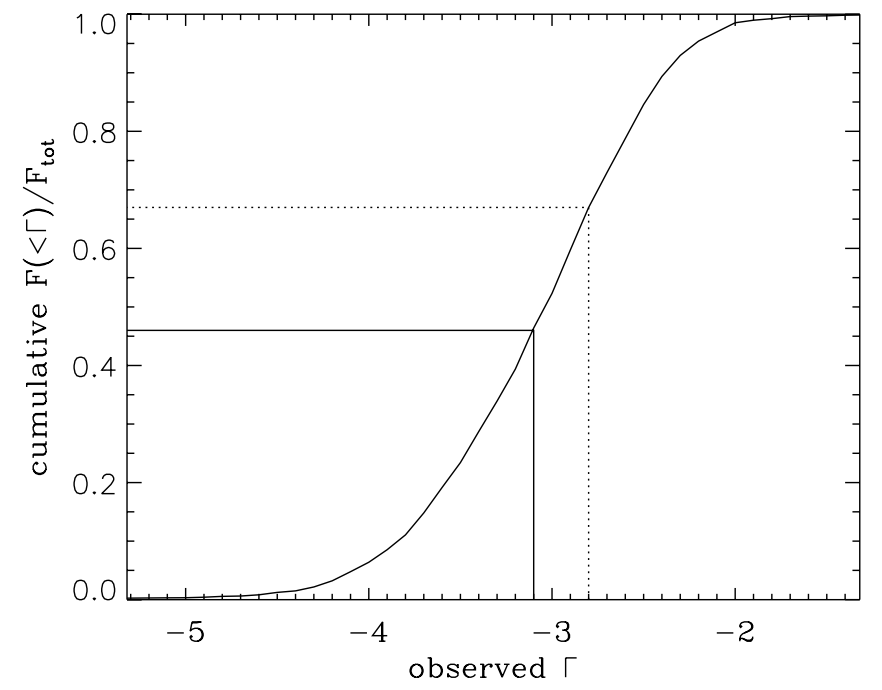

FIG. 10.-Plot of the cumulative flux between 4.2 and $6 \mathrm{keV}$ vs. the best-fit power-law index as found in Fig. 6 . The solid line shows that $46 \%$ of the flux has a power-law index steeper than -3.1 . The dotted line shows that $33 \%$ of the flux has a power law flatter than -2.8 ; the power-law index associated with a thermal bremsstrahlung model with $k T=3.5 \mathrm{keV}$.
TABLE 1

Results of the Spectral Fit to the Featureless Filament

\begin{tabular}{|c|c|}
\hline Parameter & Value \\
\hline$n_{\mathrm{H}}\left(\mathrm{cm}^{-2}\right) \ldots \ldots \ldots$ & $1.67_{-0.03}^{+0.03} \times 10^{22}$ \\
\hline 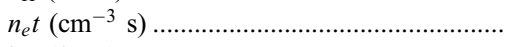 & $8.6_{-1.0}^{+1.0} \times 10^{10}$ \\
\hline kT $(\mathrm{keV}) \ldots \ldots \ldots \ldots . . . .$. & $1.8_{-0.15}^{+0.15}$ \\
\hline$\Gamma \ldots \ldots \ldots \ldots \ldots \ldots \ldots \ldots \ldots$ & $-2.09_{-0.07}^{+0.07}$ \\
\hline$F_{\text {pow }} / F_{\text {nei }}(E \in[0.4 \mathrm{keV}, 8 \mathrm{keV}]) \ldots \ldots \ldots \ldots \ldots \ldots \ldots$ & 2.23 \\
\hline 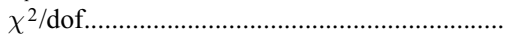 & $283 / 145$ \\
\hline
\end{tabular}

Notes.-Previous studies (Keohane 1996; Willingale et al. 2002) show that the absorption in the west side is higher than in the east, with values up to $1.7 \times 10^{22} \mathrm{~cm}^{-2}$. So a value of $1.67 \times 10^{22} \mathrm{~cm}^{-2}$ is still within the expectations.

from less flux from shock-heated plasma) also in a low-ionization timescale, $n_{e} t$. With this in mind, we extracted spectra from one of the featureless filaments located at the reverse shock. This is the same filament as that used by Hughes et al. (2000; filament "D"). We extracted the spectrum of this region in the individual event files of the megasecond image and added them with the addspec tool of ftools in HEASOFT. We fit the resulting spectrum with a model consisting of a power law and the nonequilibrium ionization (NEI) model of SPEX (Kaastra et al. 1996). The best-fit parameters are listed in Table 1.

\section{DISCUSSION}

Using a combination of deprojection and characterization of the X-ray continuum power-law slope of the $1 \mathrm{Ms}$ Chandra observation, we have determined the location of the nonthermal $\mathrm{X}$-ray emission of Cas A. We have found that a significant fraction of the X-ray continuum emission is coming from two separate rings; one can be identified with the forward shock, the other with the shell dominated by bright ejecta. About $54 \%$ of all continuum emission in the $4.2-6 \mathrm{keV}$ band is likely to be nonthermal.

The nonthermal emission from the forward shock is likely due to synchrotron radiation from electrons accelerated at the forward shock, confined to the forward shock region by synchrotron cooling (Vink \& Laming 2003; Berezhko \& Völk 2004). However, the nonthermal emission of Cas A as a whole is dominated by the contribution of the inner ring. This inner region of emission is shifted to the west of the remnant by $\sim 15^{\prime \prime}$. A similar shift was found for the location of the reverse shock by Reed et al. (1995) and Gotthelf et al. (2001) on the basis of optical data and a deprojection of radio data and a $50 \mathrm{ks}$ Chandra observation, in the Si band. Gotthelf et al. (2001) also found marginal evidence for a higher emissivity at the reverse shock in the $4-6 \mathrm{keV}$ band using the $50 \mathrm{ks}$ Chandra observation.

\subsection{Nature and Location of the Inner Nonthermal Emission}

Since some of the spectra in the west hardly show any line emission, we think it is likely that this is synchrotron emission, since nonthermal bremsstrahlung without line emission is hard to establish, unless one has peculiar abundances. Such a bremsstrahlung model was once invoked for SN 1006 (Hamilton et al. 1986) but has now been abandoned in favor of synchrotron emission. Furthermore, a recent paper by Vink (2008) shows that electrons with energies close to the thermal electron energy distribution lose their energy relatively fast due to Coulomb losses. This process is already important in the Chandra energy band for $n_{e} t<8.6 \times$ $10^{10} \mathrm{~cm}^{-3} \mathrm{~s}$, the value reported here for a filament dominated by continuum emission in the west $(\S 4.1$, Table 1$)$. For $n_{e} t \sim$ $10^{11} \mathrm{~cm}^{-3} \mathrm{~s}$, typical for Cas A (Willingale et al. 2002), one only 
expects to see nonthermal bremsstrahlung for photon energies $\gtrsim 100 \mathrm{keV}$.

When it comes to the location of the synchrotron emission, there are two possibilities: the reverse shock and the contact discontinuity. The contact discontinuity marks the border between shocked ejecta and shocked circumstellar medium. Hydrodynamical solutions show that the density and the magnetic field peak at this radius (Chevalier 1982; Lyutikov \& Pohl 2004). For a supernova remnant evolving in a stellar wind, the contact discontinuity is close to the reverse shock. So from our deprojections it is difficult to judge whether the X-ray synchrotron emission is coming from the reverse shock or the contact discontinuity. It is unlikely that electrons are accelerated to $\mathrm{TeV}$ energies at the contact discontinuity, since no viable acceleration mechanism is known (however, see Lyutikov \& Pohl 2004), but there are two ways of generating X-ray synchrotron emission at the contact discontinuity: (1) Due to an increase of the magnetic field, electrons with relatively low energies suddenly light up in X-rays. (2) High-energy electrons and positrons are created through the decay of charged pions $\left(\pi^{ \pm}\right)$, caused by hadronic cosmic-ray collisions (Gaisser 1990). Charged pions decay into muons and muon neutrinos. The muons decay into electrons/positrons and electron and muon neutrinos. The electrons and positrons thus created are often called secondary electrons and positrons.

Option 1.- For synchrotron radiation the relation between electron energy and photon energy is

$$
E_{\mathrm{ph}}=19 E_{\mathrm{TeV}}^{2} B_{\mathrm{G}} \mathrm{keV}
$$

(Ginzburg \& Syrovatskii 1965). For electrons to be invisible in $\mathrm{X}$-ray synchrotron radiation, the peak energy should be an order of magnitude lower than the X-ray continuum at 4-6 keV. Therefore, the magnetic field should increase an order of magnitude at the contact discontinuity. As an example, an electron, accelerated at the forward shock, with an energy of $3 \mathrm{TeV}$ in the typical magnetic field of Cas A of $0.5 \mathrm{mG}$, typically emits photons of $0.09 \mathrm{keV}$. When this electron suddenly enters a region of $5 \mathrm{mG}$, it will emit photons of typically $1 \mathrm{keV}$, detectable in X-rays. However, the synchrotron loss time for such an electron in a $0.5 \mathrm{mG}$ magnetic field, 18 years, is rather short compared to the age of Cas A. In this time the particle has to diffuse from the forward shock region to the contact discontinuity, which is about $0.5 \mathrm{pc}$, for a distance to Cas A of $3.4 \mathrm{kpc}$ (Reed et al. 1995). For $3 \mathrm{TeV}$ and $B=0.5 \mathrm{mG}$ the diffusion constant is $D=\eta 2 \times 10^{23} \mathrm{~cm}^{2} \mathrm{~s}^{-1}$, with $\eta=1$ corresponding to Bohm diffusion (Malkov \& Drury 2001). In $18 \mathrm{yr}$ a particle can diffuse by $R \approx(2 D t)^{1 / 2}=5 \times 10^{-3}(\eta)^{1 / 2} \mathrm{pc}$. In other words, this model only works if the magnetic field turbulence is very low, corresponding to $\eta>10,000$. This is unlikely, since the ample presence of cosmic rays results in magnetic field turbulence. Moreover, at the shock front, $\eta \sim 1$ (Vink 2006; Stage et al. 2006). For low values of $\eta$, the diffusion length $\left(l_{\text {diff }}\right.$ the length for which advection dominates over diffusion), is short: $l_{\text {diff }}=\eta 4.5 \times 10^{-4} \mathrm{pc}$. So, for low $\eta$, the advection velocity $(u=$ $v_{\mathrm{fs}} / 4$, with $v_{\mathrm{fs}}$ the forward shock velocity) is the relevant velocity. For a $v_{\mathrm{fs}}$ of $5800 \mathrm{~km} \mathrm{~s}^{-1}, u=1450 \mathrm{~km} \mathrm{~s}^{-1}$. Using this velocity, it takes 674 years to go from the forward shock to the contact discontinuity, longer than the synchrotron loss time of 18 years.

It has been argued that the magnetic field at the shock front is high but rapidly decays toward the inside (Pohl et al. 2005), increasing the synchrotron loss times and increasing the diffusion constant. However, the decay in magnetic field should be reflected in the radio emissivity from the forward shock to the inside, which is contrary to observations that show gradual increase in emissivity toward the center starting at the shock front (Gotthelf et al. 2001).

Option 2 (secondary electrons). - If the X-ray synchrotron emission from the inside is due to secondary electrons, this would be an important discovery. It would be evidence for the presence of $\mathrm{TeV}$ ion cosmic rays. Neutral pions $\left(\pi^{0}\right)$ are made in comparable quantities to charged pions. The power in secondary electrons should therefore be comparable to the luminosity in pion decay. In order to compare the fluxes from X-ray synchrotron and $\gamma$-ray radiation, one should take into account the conversion from pion energy $\left(\pi^{0} \& \pi^{ \pm}\right)$to photon energies, both in the TeV band (through $\pi^{0}$ decay) and in the X-ray band, due to synchrotron radiation from secondary electrons. Taking into account these various decay channels, one finds that $E_{\mathrm{ph}} \sim 20 E_{\gamma \mathrm{TeV}}^{2} B_{\mathrm{G}} \mathrm{keV},{ }^{5}$ so the X-ray synchrotron flux above $4 \mathrm{keV}$ should correspond to roughly the $\gamma$-ray flux above $20 \mathrm{TeV}$. For the X-ray synchrotron flux above $4 \mathrm{keV}$ we found $2.7 \times 10^{-10} \mathrm{erg} \mathrm{s}^{-1} \mathrm{~cm}^{-2}$. This is a factor of 130 higher than the $\gamma$-ray flux above $1 \mathrm{TeV}$, which we calculated to be $2.1 \times$ $10^{-12} \mathrm{erg} \mathrm{cm}^{-2} \mathrm{~s}^{-1}$ using the photon flux and photon index above $1 \mathrm{TeV}$ reported by the MAGIC collaboration (Albert et al. 2007). Since we should actually have evaluated the flux above $20 \mathrm{TeV}$, we can rule out that the $\mathrm{X}$-ray synchrotron emission is caused by secondary electrons.

In our view it is therefore most likely that the X-ray synchrotron emission is caused by electrons accelerated at the reverse shock. Similarly to the forward shock region the electrons are likely to be confined to a region near the shock itself. For a long time the reverse shock as location for acceleration has been neglected because of its putative low magnetic field. Moreover, the abundance pattern of cosmic rays is consistent with acceleration from plasmas with solar abundances (Hörandel 2008). As far as the magnetic field is concerned, however, Ellison et al. (2005) have argued that if magnetic field amplification (Bell \& Lucek 2001) works at the forward shock, it is likely to operate at the reverse shock as well. Also, for the SNR RCW 86 it has been suggested that the X-ray synchrotron emission in the southwest of the remnant is coming from electrons accelerated at the reverse shock (Rho et al. 2002).

\subsection{Reverse Shock Velocity in the West and the Presence of Synchrotron Emission}

The presence of X-ray synchrotron radiation from shockaccelerated electrons is only expected if the shock velocity is high enough; shock acceleration theory predicts for the maximum photon energies (Aharonian \& Atoyan 1999)

$$
E_{\mathrm{ph}}=0.5 \eta^{-1}\left(\frac{v_{s}}{2000 \mathrm{~km} \mathrm{~s}^{-1}}\right)^{2} \mathrm{keV}
$$

where $v_{s}$ is the shock velocity. Note that the photon energy is independent of the magnetic field. For the reverse shock the velocity in equation (4) refers to the shock speed in the frame of the ejecta. The ejecta velocity is equal to the free expansion velocity $v_{f, \text { ej }}=$ $r_{\mathrm{ej}} / t$. For the reverse shock the shock velocity as seen by the ejecta is therefore $v_{s, \text { ej }}=v_{f, \text { ej }}-v_{s, \text { obs }}$, with $v_{s, \text { obs }}$ the shock velocity in

\footnotetext{
5 The decay product of $\pi^{0}$ decay is two photons, with each, on average, an energy $E_{\gamma \mathrm{TeV}}=0.5 E_{\pi^{0}}$. For the charged pions, $\pi^{ \pm}$, the final decay product consists of electrons (positrons) and neutrinos $\left[\pi^{ \pm} \rightarrow \mu^{ \pm}+\nu_{\mu}\left(\bar{\nu}_{\mu}\right), \mu^{ \pm} \rightarrow e^{ \pm}+\nu_{e}\left(\bar{\nu}_{e}\right)+\right.$ $\left.\nu_{\mu}\left(\bar{\nu}_{\mu}\right)\right]$. In this case the final electron or positron also take up, on average, half the initial pion energy $E_{e^{ \pm}} \approx 0.5 E_{\pi^{ \pm}}$; hence, for a given pion energy, $E_{e^{ \pm}} \approx E_{\gamma \mathrm{TeV}}$. This electron or positron emits synchrotron radiation at a peak frequency given by eq. (3). Hence, the close resemblance of this equation with eq. (3).
} 
the frame of the observer. The presence of X-ray synchrotron radiation from the reverse shock in the western half of Cas A suggests a higher reverse shock than in the rest of the remnant. Proper motions of knots at the inside of Cas A in X-rays were most recently measured by DeLaney et al. (2004), but some details of the measurements, including measurements of the proper motions as a function of azimuth, only appeared in Appendix 4.4 of Delaney (2004, Fig. 4.6). In this figure, we see that in the west the expansion rate is between $-0.1 \%$ and $0.1 \% \mathrm{yr}^{-1}$; this corresponds to $v_{s, \mathrm{obs}}$ at the reverse shock of approximately -2000 to $2000 \mathrm{~km} \mathrm{~s}^{-1}$, implying a shock velocity ${ }^{6} v_{s, \text { ej }} \approx 3900-7900 \mathrm{~km} \mathrm{~s}^{-1}$. In the northern and eastern parts of Cas A, Delaney (2004) finds expansion rates of $0.2 \% \mathrm{yr}^{-1}$, corresponding to $v_{s, \text { obs }} \approx 4000 \mathrm{~km} \mathrm{~s}^{-1}$ and a shock velocity of $v_{s, \text { ej }} \approx 1900 \mathrm{~km} \mathrm{~s}^{-1}$ at the reverse shock.

In the optical Morse et al. (2004) report a shock velocity of $v_{s, \text { ej }} \approx$ $3000 \mathrm{~km} \mathrm{~s}^{-1}$ for a filament in the northwest. In our maps (Fig. 6, left) we find that this region has a hard power-law slope, suggesting the presence of synchrotron radiation, but the overall X-ray emission is dominated by thermal X-ray line emission (Fig. 6, right).

It indeed looks like the presence of X-ray synchrotron emission from the inner ring corresponds with reverse shock velocities in excess of $2000 \mathrm{~km} \mathrm{~s}^{-1}$. To some extend this is surprising, since the X-ray synchrotron emissivity function is rather broad and can result in some synchrotron emission beyond the maximum photon energy as defined in equation (4). On the other hand, $\eta=1$ corresponds to Bohm diffusion and represents the case for maximum acceleration efficiency. The fact that $2000 \mathrm{~km} \mathrm{~s}^{-1}$ seems close to the velocity dividing the presence or absence of X-ray synchrotron radiation again suggests that acceleration takes place close to the Bohm limit. Equation (4) suggests that the spectra in the west of the remnant will be harder than in the rest, where $v_{s, \text { ej }}$ is lower.

Note that the high reverse shock velocities in the west (and low observed velocities) are not in agreement with analytic hydrodynamic solutions for an SNR evolving in a stellar wind (Laming \& Hwang 2003). Adapting the parameters of this model such that the forward shock radius and velocity and the reverse shock radius match those of Cas A, we find $v_{s}$, ej ranging between 1600 and

\footnotetext{
${ }^{6}$ We have checked this result using the Chandra 1 Ms observation and an observation of Chandra made in 2000 using the procedure described in Vink et al. (1998). We concentrated on filament D. The result confirms the lower expansion or even backward velocities: we found $-970 \pm 140 \mathrm{~km} \mathrm{~s}^{-1}$, i.e., the filament seems to move toward the center. This implies $v_{s, \text { ej }} \approx 6900 \mathrm{~km} \mathrm{~s}^{-1}$. Vink et al. (1998) already reported a lower expansion for the whole western part of Cas A.
}

$2300 \mathrm{~km} \mathrm{~s}^{-1}$, significantly lower than in the west, but in agreement with those in the eastern part of Cas A. This suggests that the circumstellar density structure is more complex in the west.

\section{CONCLUSIONS}

We have presented an analysis of the spatial and spectral variation of the X-ray continuum emission of Cas A, based on the $1 \mathrm{Ms}$ Chandra observation. We find that harder continuum spectra are associated with the filaments, dominated by continuum emission, suggesting that the harder spectra are caused by nonthermal radiation. A dominant fraction of the nonthermal emission appears to come from the reverse shock region. We have discussed various options for the nature of the nonthermal emission and its origins. Some of our conclusions were also independently obtained by Uchiyama \& Aharonian (2008) but based on X-ray variability. Based on our analysis and discussion, we come to the following conclusions:

1. The power-law index of the spectrum between 4.2 and $6.0 \mathrm{keV}$ is an indicator of $\mathrm{X}$-ray synchrotron emission; there is a correlation between filaments, dominated by continuum emission and hard spectra.

2. Hard X-ray spectra are not exclusively associated with filaments, dominated by continuum emission, suggesting that nonthermal emission also comes from other regions.

3. The nonthermal X-ray emission is likely to be synchrotron radiation.

4. The nonthermal emission accounts for about $54 \%$ of the overall continuum emission in the $4-6 \mathrm{keV}$ band.

5 . In the western part of Cas A, most X-ray synchrotron emission comes from the reverse shock.

6. The dominance of X-ray synchrotron emission from the west is probably the result of a locally higher reverse shock velocity of $v_{s} \sim 6000 \mathrm{~km} \mathrm{~s}^{-1}$ (corresponding to a lower proper motion) than in the eastern region $\left(v_{s} \sim 1900 \mathrm{~km} \mathrm{~s}^{-1}\right)$.

We want to thank Tracey DeLaney for providing us with a recent radio map of Cas A. We would like to thank Frank Verbunt for carefully reading the manuscript and Michele Cappellari for the software we used to display some of our results. This work is supported by the NWO-VIDI grant of J. V.

Facilities: CXO (ACIS).
Aharonian, F. A., \& Atoyan, A. M. 1999, A\&A, 351, 330

Aharonian, F., et al. 2001, A\&A, 370, 112 2004, Nature, 432, 75

Albert, J., et al. 2007, A\&A, 474, 937

Allen, G., Gotthelf, E. V., \& Petre, R. 1999, in Proc. 3rd Int. Cosmic Ray Conf. (Salt Lake City), 480

Allen, G. E., et al. 1997, ApJ, 487, L97

Bell, A. R., \& Lucek, S. G. 2001, MNRAS, 321, 433

Berezhko, E. G., \& Völk, H. J. 2004, A\&A, 419, L27

Bleeker, J. A. M., et al. 2001, A\&A, 365, L225

Chevalier, R. A. 1982, ApJ, 258, 790

DeLaney, T., et al. 2004, ApJ, 613, 343

. 2005, in X-Ray and Radio Connections, ed. L. O. Sjouwerman \& K.K

Dyer, http://www.aoc.nrao.edu/events/xraydio

Delaney, T. A. 2004, Ph.D. thesis, Univ. Minnesota

Ellison, D. C., Decourchelle, A., \& Ballet, J. 2005, A\&A, 429, 569

Favata, F., et al. 1997, A\&A, 324, L49

Gaisser, T. K. 1990, Cosmic Rays and Particle Physics (Cambridge: Cambridge

Univ. Press)

Ginzburg, V. L., \& Syrovatskii, S. I. 1965, ARA\&A, 3, 297

Gotthelf, E. V., et al. 2001, ApJ, 552, L39

\section{REFERENCES}

Green, D. A., Reynolds, S. P., Borkowski, K. J., Hwang, U., Harrus, I., \& Petre, R. 2008, MNRAS, 387, L54

Hamilton, A. J. S., Sarazin, C. L., \& Szymkowiak, A. E. 1986, ApJ, 300, 698

Hörandel, J. R. 2008, Adv. Space Res., 41, 442

Hughes, J. P., Rakowski, C. E., Burrows, D. N., \& Slane, P. O. 2000, ApJ, 528, L109

Hwang, U., et al. 2004, ApJ, 615, L117

Kaastra, J. S., Mewe, R., \& Nieuwenhuijzen, H. 1996, in Proc. 11th Colloq. on UV and X-Ray Spectroscopy of Astrophysical and Laboratory Plasmas, ed. K. Yamashita \& T. Watanabe (Tokyo: Universal Academy Press), 411

Keohane, J. W. 1996, in ASP Conf. Ser. 99, Cosmic Abundances, ed. S. S. Holt \& G. Sonneborn (San Francisco: ASP), 362

Koyama, K., et al. 1995, Nature, 378, 255

Laming, J. M. 2001, ApJ, 546, 1149

Laming, J. M., \& Hwang, U. 2003, ApJ, 597, 347

Landsman, W. B. 1993, in ASP Conf. Ser. 52, Astronomical Data Analysis Software and Systems II, ed. R. J. Hanisch, R. J. V. Brissenden, \& J. Barnes (San Francisco: ASP), 246

Lucy, L. B. 1974, AJ, 79, 745

Lyutikov, M., \& Pohl, M. 2004, ApJ, 609, 785

Malkov, M. A., \& Drury, L. 2001, Rep. Prog. Phys., 64, 429 
Morse, J. A., et al. 2004, ApJ, 614, 727

Pohl, M., Yan, H., \& Lazarian, A. 2005, ApJ, 626, L101

Reed, J. E., Hester, J. J., Fabian, A. C., \& Winkler, P. F. 1995, ApJ, 440, 706

Renaud, M., et al. 2006, ApJ, 647, L41

Reynolds, S. P., Borkowski, K. J., Green, D. A., Hwang, U., Harrus, I., \& Petre, R. 2008, ApJ, 680, L41

Rho, J., Dyer, K. K., Borkowski, K. J., \& Reynolds, S. P. 2002, ApJ, 581, 1116

Rothschild, R. E., \& Lingenfelter, R. E. 2003, ApJ, 582, 257

Stage, M. D., Allen, G. E., Houck, J. C., \& Davis, J. E. 2006, Nature Physics, 2, 614

The, L.-S., et al. 1996, A\&AS, 120, C357

Thorstensen, J. R., Fesen, R. A., \& van den Bergh, S. 2001, AJ, 122, 297

Uchiyama, Y., \& Aharonian, F. A. 2008, ApJ, 677, L105
Vink, J. 2006, preprint (astro-ph/0601093)

$$
\text { 2008, A\&A, 486, } 837
$$

Vink, J., Bloemen, H., Kaastra, J. S., \& Bleeker, J. A. M. 1998, A\&A, 339, 201

Vink, J., Kaastra, J. S., \& Bleeker, J. A. M. 1996, A\&A, 307, L41

Vink, J., \& Laming, J. M. 2003, ApJ, 584, 758

Vink, J., et al. 1999, A\&A, 344, 289

2001, ApJ, 560, L79

Willingale, R., Bleeker, J. A. M., van der Heyden, K. J., Kaastra, J. S., \& Vink, J. 2002, A\&A, 381, 1039

Willingale, R., West, R. G., Pye, J. P., \& Stewart, G. C. 1996, MNRAS, 278, 749

Yang, X.-J., Lu, F.-j., \& Chen, L. 2007, preprint (arXiv: 0712.1071)

Zirakashvili, V. N., \& Aharonian, F. 2007, A\&A, 465, 695 